\title{
Efficiency of Neutron Tomography in visualizing the internal structure of metal artefacts from Mapungubwe Museum Collection with the aim of Conservation
}

Farahnaz. Koleini ${ }^{1 *}$, Frikkie. de Beer ${ }^{2}$, M.H.Alex. Schoeman ${ }^{3}$, Innocent. Pikirayi ${ }^{4}$,

Shadreck. Chirikure ${ }^{5}$, Gawie. Nothnagel ${ }^{6}$, Jacob.Mabuti. Radebe ${ }^{7}$,

Aim: Estimating physical stability as well as revealing signs of fabrication in metal artefacts via two complementary non- destructive methods, neutron- and X-ray tomography were the main goals of this study.

\section{Abstract:}

To aid conservation management of metal artefacts improved knowledge of the internal structure and degree of corrosion therein is highly desired. In this study, neutron- and X-ray tomography was chosen as two complementary non invasive visualization techniques to study internal structure and corrosion of five representative examples of archaeological metal artefacts from the Mapungubwe museum collection. Tomography was performed at the Neutron Radiography (SANRAD) facility of the South African Nuclear Energy Corporation where thermal neutrons as well as complementary X-rays are conveniently available under one roof. Comparative studies revealed neutron tomography to be generally superior to X-ray tomography due to the enhanced penetration properties of neutrons through the metal objects. In this paper the rich capability of neutron tomography as a non-destructive visualization aid for scientific conservation purposes is introduced and supported by results achieved for the five selected real artefacts.

Keywords: neutron tomography/ X-ray/ archaeological metal/ conservation/ corrosion percentage/ Mapungubwe

\footnotetext{
1 - Department of Anthropology and Archaeology, University of Pretoria, South Africa

2 -Radiation Science, Necsa, PO box 582, Pretoria, South Africa

3 - Department of Archaeology, University of the Witwatersrand, South Africa

${ }^{4}$-Department of Anthropology and Archaeology, University of Pretoria, South Africa

5 -Department of Archaeology, University of Cape Town, South Africa

${ }^{6}$-Necsa, PO box 582, Pretoria, South Africa

7 -Radiation Science, Necsa, PO box 582, Pretoria, South Africa
} 


\section{Introduction}

Conservation of archaeological collections is a stepwise process, which starts with a visual study of the objects. This kind of study is not restricted to the surface and extends toward internal structure of the artefact and entails gathering of information about method of fabrication, usage, physical stability as well as signs of restoration that may have been previously attempted during the life time of the object. Data of this nature allows a conservator to take an informed decision regarding expected survival of the object and associated needs for conservation intervention. Known investigative technologies such as the binocular microscope, infrared and ultraviolet light illumination assist greatly with enhanced perception of surface features. The advent of penetrating radiation techniques such as X-rays, gamma-rays and neutrons added yet another powerful string to the bow of the conservator in that these rays make it possible to non-destructively visualize aspects of internal structures of artefacts. These so-called non-destructive examination (NDE) techniques are utilized to different degrees based on their accessibility, intrinsic capability and efficiency in yielding the desired information.

The South African radiography and tomography (SANRAD) facility that is located at the SAFARI-1 nuclear research reactor based at Necsa, near Pretoria, provided an ideal opportunity to use thermal neutrons and X-rays to perform NDE of internal structures of selected metal artefacts from the Mapungubwe museum collection. Mapungubwe is an important Iron Age site in the north of South Africa at the Shashi Limpopo confluence area [1]. A huge number of metal artifacts that date from 1220 $\mathrm{AD}$ to $1290 \mathrm{AD}$ were found by archaeologists during past excavation from 1933-2000. Except for gold jewelries and ornaments, there are a large number of copper and iron trinkets and implements that are kept in storage at the Mapungubwe museum of the University of Pretoria in different states of preservation from low to heavily corroded. Some of these became completely disintegrated and converted to a bulk of corrosion products thus creating an impetus to start a scientific conservation process.

Estimation of (remaining) metal core size, its physical shape and continuity along the corroded metalwork, the position, percentage and penetration character of corrosion products, the presence of defects such as cracks or voids as well as the remaining 
evidence of fabrication methods were the main goals of this study. This paper compares the capability of neutrons relative to X-rays to provide the visualisation results in order to acquire the goals of the study. Advantages of tomography above two-dimensional conventional radiography will also be shown.

\section{X-Ray- and neutron attenuation and imaging characteristics}

Imaging of the complete three dimensional (3D) object becomes possible with penetrating radiation. To form a proper visualization of the interior of the object two conditions need to be fulfilled. The first is that the radiation must be able to pass through the interior. The second is that, whilst doing so, sufficient interaction on different scales with structures in the interior of the object must occur to provide sufficient contrast to enable visualization. This means that the radiation must be able to penetrate the object but that the object must not be fully transparent to the radiation. This condition is used to perform some quantitative estimates of the imaging power of $\mathrm{X}$-rays and neutrons respectively for different types of materials.

Equation (1) below describes attenuation for the special case where a parallel beam with intensity $I_{0}$ impinges upon a slab of material. It provides a formula for the remaining intensity $I$ after penetration through a thickness $x(\mathrm{~cm})$ of material of density $\rho\left(\mathrm{g} / \mathrm{cm}^{3}\right)$ and mass attenuation coefficient $\mu\left(\mathrm{cm}^{2} / \mathrm{g}\right)$.

$$
I=I_{0} \exp (-\mu \rho x)
$$

Although strictly speaking accurate only for parallel geometry, the equation can be used to estimate depth of penetration into real objects with more complicated geometry. It shows that the intensity drops off exponentially with distance into the object and that the factors influencing the attenuation of the incident radiation beam are the attenuation coefficient (radiation type dependent ) and the material density and the thickness (object dependent only)[2]. The same general relation applies for X-rays and neutrons, the only difference being in the mass absorption properties ( $\mu$-values) for interaction of X-rays and neutrons with the different kinds of materials in the object. This depends on the fundamental properties of the two types of radiation. X- 
rays interact with the electron cloud around the atom and neutrons interact with the nuclei [3].

Equation (1) can be rearranged to explicitly estimate the thickness of material, which will attenuate the original intensity $I_{0}$ by $99 \%$, say. The resultant formula is:

$x=\frac{4.6}{\rho \mu} \mathrm{cm}$

Only $1 \%$ of the radiation manages to penetrate deeper than this value and one can thus safely assume that this constitutes a good estimate for "absolute" visualization depth.

For X-rays there exist a direct relation between the mass attenuation coefficient and the effective atomic number of an element or compound, also known as Moseley's law. The high atomic number of heavy metals thus result in them having larger $\mu$ values, which in combination with their high densities ( $\rho$-values) limits penetration only to the surface regions of the objects as predicted by equation (2). This is especially true for low energy $X$-rays $(<100 \mathrm{kV})$. For instance for $60 \mathrm{keV} X$-rays on iron, $\rho=7.86 \mathrm{~g} . \mathrm{cm}^{-3}, \mu=1.2 \mathrm{~cm}^{2} \cdot \mathrm{g}^{-1}$ and thus $x \doteq 0.5 \mathrm{~cm}$. Objects with characteristic size larger than this become opaque to the X-ray beam and cannot be visualized in their interior. The opposite extreme of low-Z materials and low density predicts "transparent objects" that again leads to ineffective or negligible visualization capability. X-rays are therefore generally not well suited to study objects exclusively containing materials made of light elements, or large objects contain heavy metals such as iron, lead, copper, silver, gold, etc. The latter are of course materials of choice for artifact construction.

Nowadays beside conventional X-ray radiography other techniques such as Industrial high density tomography (CT) [4] and computerized axial tomography (CAT) [5] are used to study metal objects more efficiently due to more sensitive detection, higher energy X-ray source $(420 \mathrm{kV})$ and higher resolution detectors. They are capable of scanning larger, high density objects in order to reconstruct accurate threedimensional virtual digital images thereof [4]. However, taking into consideration that most radiography facilities do not have such a powerful X-ray source, the dense 
material like silver, iron, copper and their alloys appear opaque to X-rays and proper imaging of thick metal objects become severely compromised.

Table 1

Linear attenuation coefficients of some metals and element constituent of corrosion products for thermal neutrons and $125 \mathrm{kV} \mathrm{X}$-rays

\begin{tabular}{lllllllllll}
\hline Element & $\mathrm{H}$ & $\mathrm{O}$ & $\mathrm{Cl}$ & $\mathrm{Fe}$ & $\mathrm{Cu}$ & $\mathrm{Zn}$ & $\mathrm{Ag}$ & $\mathrm{Sn}$ & $\mathrm{Au}$ & $\mathrm{Pb}$ \\
\hline Atomic mass & 1.0 & 15.9 & 35.4 & 55.8 & 63.5 & 65.3 & 107.8 & 118.7 & 196.9 & 207.2 \\
\hline X-rays $\boldsymbol{\mu}\left(\mathbf{c m}^{-1}\right)$ & 0.02 & 0.16 & 0.23 & 1.57 & 1.97 & 1.64 & 5.67 & 3.98 & 35.94 & 22.81 \\
$\begin{array}{l}\text { Thermal neutrons } \\
\text { i }\left(\mathbf{c m}^{-1}\right)\end{array}$ & 3.44 & 0.17 & 1.33 & 1.19 & 1.07 & 0.35 & 4.04 & 0.21 & 6.23 & 0.38 \\
\hline
\end{tabular}

Source: http://physics.nist.gov/physRefData/XrayMassCoef/tab3.html and http://www.ncnr.nist.gov/resource/n-lenghts/list.html

Fortunately neutrons afford a means to study these objects. Neutron attenuation shows no systematic relationship with atomic number, such as with X-rays (See Table 1). In particular neutrons can penetrate the heavy metals readily and can also visualize material containing low atomic number elements such as $\mathrm{H}, \mathrm{B}, \mathrm{C}, \mathrm{O}, \mathrm{Al}$, etc. These properties of the neutrons in radiography make it possible to detect organic materials inside metal objects, a feat that cannot be achieved with X-rays. This becomes of particular interest to conservation management as the majority of corrosion products have hydrogen in their composition. At the same time the high penetration ability of neutrons through metal objects renders it a suitable technique in retracing fabrication methods in archaeological metal artefacts.

The first study in utilization of neutron radiation for radiography in archaeology was submitted in 1996 by Schillinger [6]. Thereafter, many research projects were conducted that exploits the unique non-destructive visualization powers of neutrons (complementary with those of X-rays) through digital radiography and tomography to reveal new details about technology of production and state of degradation of objects also for the science of conservation of historical objects [7-11]. 


\section{Experimental methods}

\subsection{Instrumentation}

Neutron/X-ray Radiography and Tomography studies of the metal objects from the Mapungupwe collection at UP were performed at the SANRAD facility, which is located on the beam port floor of the SAFARI-1 nuclear research reactor at beam port No. 2. The neutron and gamma beams produced by the fission reaction in SAFARI-1 are filtered by a Bismuth crystal and the scattered neutrons and gamma rays are collimated towards the sample and detector. The result is a $93 \%$ thermal neutron beam that passes onto and through the sample. A $100 \mathrm{kV}$ X-ray tube can be installed at the exit of the collimator to replace the neutron beam delivery with X-rays. It affords the opportunity to use and compare the result of two penetrating radiation types in the study of metal objects.

The samples were positioned on a rotary table between the radiation source and the scintillator screen detector. To minimize the second and higher order detrimental scattered neutrons (unwanted background) from the samples, they were placed more than $80 \mathrm{~mm}$ from the detector. For high spatial resolution results, the samples were placed as near to the detector as possible. Scintillator screens used for neutron and Xray detection are ${ }^{6} \mathrm{LiF} / \mathrm{ZnS}: \mathrm{Cu}, \mathrm{Al}, \mathrm{Au}$ and $\mathrm{Gd}_{2} \mathrm{O}_{2} \mathrm{~S}$ respectively. The optical images from the scintillator are reflected via a $45^{\circ}$ mirror onto a lens system (SMC-Pentax lenses) that focuses the image onto a Peltier-cooled CCD camera (Andor type) with a 1024 -1024 pixel array and 16-bit image output device at $2 \mu \mathrm{m} /$ pixel. The camera chip is cooled to $-45^{\circ} \mathrm{C}$ to minimize dark current and thermal noise. Projections were recorded by incremental rotation of the sample through an angular interval of, $\Delta \theta=$ $0.96^{\circ}$ from $0^{\circ}$ to $360^{\circ}$. This yields a total of 375 projections for each tomogram. Octopus reconstruction software was used to post-process the data into $2 \mathrm{D}$ axial slices. A full description of the facility can be found in reference [11].

After reconstruction a full 3-D virtual image of the object is available. VGStudioMAX visualization software allows extraction of quantitative information regarding the distribution of features in the image in any desired slice through the 
object. Those individual slices can also be visualized and analysed through open source software such as IMAGEJ which is available on the www. For proper interpretation of radiographs and tomogram slices provided later in this paper, it must be remembered that slices contain only the image information contained in the plane of the slice itself. A radiograph, on the other hand contains all the image information projected onto a plane of observation (the scintillator screen) for a particular orientation of the object in the beam.

\subsection{Selection of artefacts}

Five (5) objects of the Mapungubwe assemblage were chosen to study relative imaging capability with neutrons and X-rays. Two (2) iron and three (3) copper artefacts were selected with different thickness and shape.

\subsection{Estimation of corrosion percentage}

When there is a simple invariant or symmetry of the corrosion structure, it becomes easy to estimate volume fraction of corrosion via direct estimation of area fraction along any representative slice. In Illustration 1 (a) below we depict a cylindrical object with an annular corrosion product layer of approximately constant thickness along its length axis.

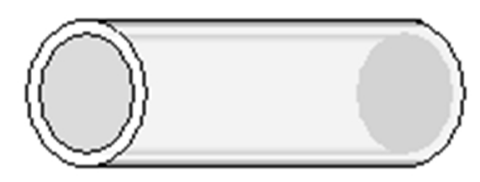

(a)

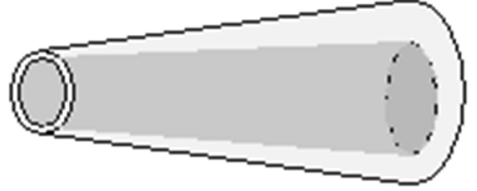

(b)

Figure 1: A schematic illustration of two corrosion scenarios 
In such a simple case (Figure.1a) any single axial slice will provide sufficient information to estimate the volume corrosion percentage of the whole object. In fact the volume percentage becomes equivalent to the area percentage in the slice:

$C \%=\frac{A_{\text {corroded }}}{A_{\text {total }}} \times 100$

By adjusting contrast and brightness (ImageJ open source analytical software) the corrosion region in the slice can be contrasted and identified more clearly to improve accuracy. In (Figure. 2) we have such a scenario for the shaft region of a real object (the spatula also shown in Figure. 3). Using the software tools it becomes possible to isolate the corroded region as shown in (Figure. 2a). The area of the corroded part as well as the total object area can then be sensitively determined. The exact units are irrelevant as we are only interested in the dimensionless ratio as defined by equation (3).
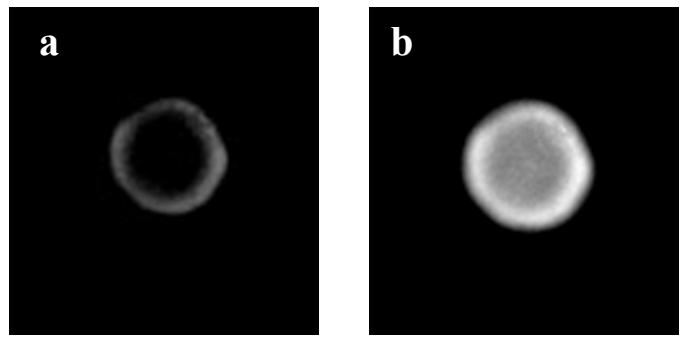

Figure 2. Slice perpendicular to the spatula shaft axis with adjusted contrast to show: (a) the corrosion ring alone; and (b) the metal core in grey and corrosion in white.

For more complicated scenarios, such as in (Figure. 1b), a single slice will not suffice to estimate volume percentage as each axial slice now clearly yield a different area fraction of corrosion. For relatively simple geometries volume fractions can still be estimated from a finite number of axial slices, say $\mathrm{N}$, of known length each, which constitutes a distance along which the corrosion properties remain constant or approximately so. The estimated average volume percentage of corrosion can then becomes:

$\% C=\frac{100}{N} \sum_{i=1}^{N} R_{i} d_{i}$

Here $R_{i}$ is the area corrosion fraction of section $i$, and $d_{i}$ is the length of that section. When a simple geometry, like the cases illustrated, does not apply the determination 
of corrosion percentage becomes difficult unless advanced image processing techniques (such as available in ImageJ) are applied to integrate slices along the object analogous to what is shown by equation (4). For percentage corrosion determination in this study ImageJ imaging techniques were applied due to the unavailability of VGStudioMAX analytical software.

\section{Investigated items and results}

\subsection{Spatula}

This small delicate iron object seems to have been used as a pendant (Figure.3a). It was found southwest of the Mapungubwe Hill (Southern Terrace) during excavation $\mathrm{K} 8$ in 1971 at a depth of $45 \mathrm{~cm}$ in a layer of loose sand that was dated to $1284 \mathrm{AD}$ [1]. Its length is $69.48 \mathrm{~mm}$ and its thickness from shaft to tip of blade is between 1.33 and 5. $29 \mathrm{~mm}$. It fractured into two parts that appears to be the result of destructive sampling in the past since the breakage surface is completely even. It has an integral long shaft that was bent to form an eye for suspension (the separate globular part). In the radiographs the edge of the shaft appears serrated. This feature, details of which are hard to distinguish by naked eye, seems to be remnants of a thin iron wire wound around the shaft that has become completely corroded, flaked and partially disintegrated. The aim of the tomographic study was to estimate the size of the remaining metal core, to distinguish some detailed features of the design and manufacture and to assess physical stability of the object.

Neutron- and X-ray tomography was performed successfully on this small sample. (Figure. 3b) shows a 2D neutron transmission radiograph of the spatula. Due to the high penetrability of neutrons through the metal it is not possible to clearly distinguish between the remaining metal core and corroded material in this image. A survey of tomogram slices, however, provides more detail about the extension of the corroded area. The particular sagittal slice near the mid plane of the object, shown in (Figure.3c), is a good illustration of this. The higher contrast region around the edge is now more visible and represents the corrosion products on the outside. The high contrast of the surface corroded area is the result of the presence of hydrogen, which has a high neutron attenuation coefficient, in the chemical composition of corrosion 

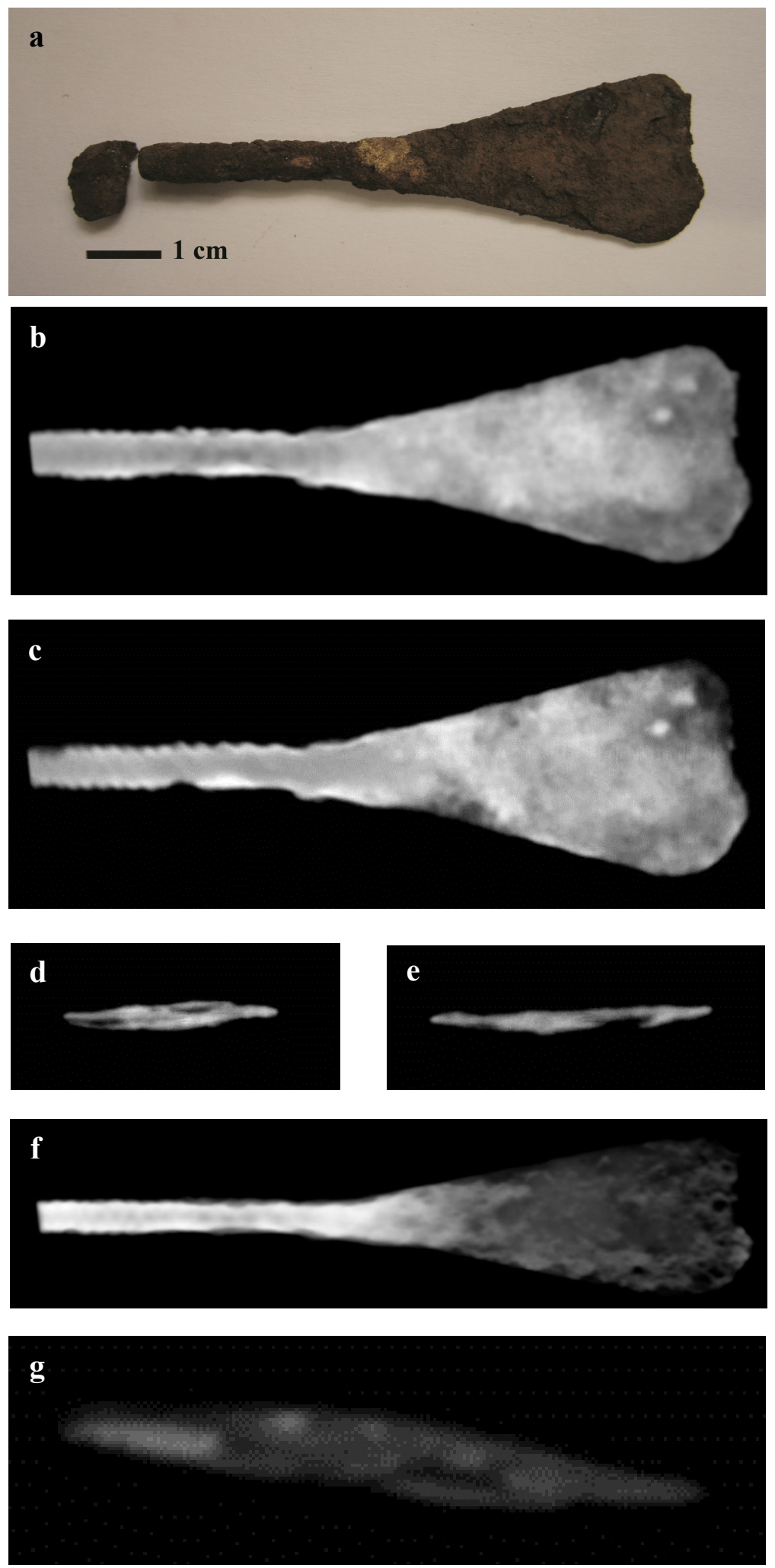

Figure 3. (a) Iron spatula from the Mapungubwe southern terrace. (b) Neutron 2D image. c) Sagittal tomogram slice near the mid plane showing detachment of corroded metal in the blade as dark areas. (d, e) Neutron tomogram axial slices illustrating lamination and flaking in the blade. (f) X-ray 2D image showing the metal core in the same zone as in the neutron sagittal slice. (g) X-ray axial slice tomogram with evidence of lamination in the blade.

blade 
products. While it remains hard to assign a specific part as belonging to intact metal, features become visible in (Figure. 3c) that may be attributed to a combination of factors such as different composition of corrosion products, lamination and flaking (darker "hollow" areas). A survey of axial slices (virtual cuts perpendicular to the length axis of the object) provides further details about the presence of flaking and lamination (Figure. $3 d$ and e). Lamination is visible as a longitudinal grey line and flaking as dark areas, indicating loss of material.

As expected for small metallic samples (see Section 2) the X-ray 2D image (Figure. 3f) reveals the metal core with much higher contrast in the shaft region than is achievable with neutrons, making the exact position of the metal core more recognizable than in the neutron image. This is the result of high interaction of X-rays with metals in general. Gradual decreasing of corrosion density toward the tip of the blade is obvious in the X-ray image. This small spatula was the only object in this assemblage where $\mathrm{X}$-ray tomography with $100 \mathrm{kV}$ energy provided better results for recognition of the remaining metal core. This is the result of relatively small corroded part (not usually seen by X-rays), high contrast of the metal part (best seen by X-rays on small objects) and the small size of the object (allowing X-ray penetration through the object).

However, the wire twisted around the shaft, is seen with greater clarity in the neutron images (Figure $3 \mathrm{~b}$ and $c$ ). The fact that the wire feature stands out much clearer in the neutron images, suggest that the original iron has become almost completely corroded. Based on neutron tomography axial slices, approximately $59 \%$ of the object consists of corrosion products, which is mostly concentrated in the blade.

\subsection{Copper ingot}

This Plano-convex (bun) ingot (Figure. $4 a$ and $b$ ) was found at Mapungubwe Hill Rock shelter in 1935. Its weight is $132.2 \mathrm{~g}$ and it is $54.50 \mathrm{~mm}$ in diameter. Its thickness is $6.90 \mathrm{~mm}$ around the border and $15.62 \mathrm{~mm}$ in the centre. The use of radiography and tomography in this case was utilized to non-destructively assess the distribution of internal voids in order to reveal more about the conditions of casting. 

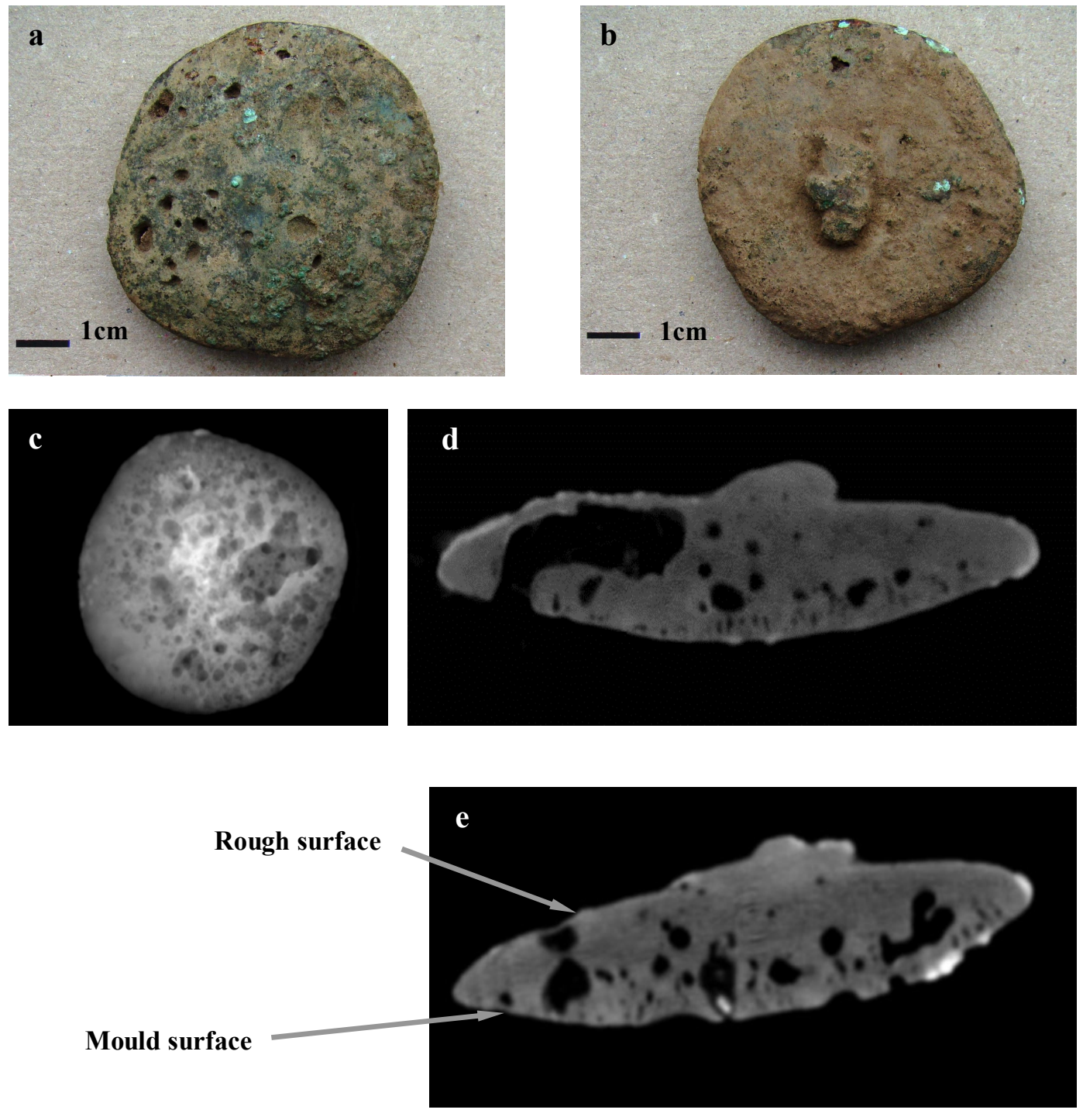

Figure 4: Copper ingot from Mapungubwe hill rock shelter. (a) Mould side surface. (b) Rough (top) surface with protrusion. (c) Neutron 2D image showing a plane projection of voids. (d) Frontal slice of the ingot showing pore distribution in the interior. (e) Axial slice showing corrosion (bright white) only on the surface and the inside of some of open voids.

X-ray radiography, because of its low penetration into the copper ingot, was now not successful in revealing the structure of internal voids, nor the presence and distribution of surface corrosion. The neutron 2D-radiograph, on the other hand, reveals the porous structure of the ingot and indicates that many gas bubbles became 
trapped during the cooling phase of the molding process. The $2 \mathrm{D}$ radiograph provides only a projection of the real $3 \mathrm{D}$ pores onto a plane and therefore may lead to ambiguous void shapes, sizes and distribution in the sample (Figure. 4c). The distribution of pores perpendicular to the plane of view of (Figure. 4c), for instance, cannot be known or derived from the radiograph. With 3D neutron tomography, however, the full three dimensional constructed image is available and the distribution of pores can be studied along any particular direction by application of appropriate image slicing.

This is nicely illustrated by the frontal slices shown in (Figure. $4 d$ and e.) These are slices perpendicular to the viewing plane of (Figure. 4c) and now allows the up-down pore distribution to be seen. It now becomes clear that a relatively small upper crust of the sample (where the protrusion is seen) appears relatively pore-free whereas numerous large pores are distributed throughout the interior elsewhere. The pores are characteristic of trapped gas accumulation and are known to occur under given conditions of casting. In fact, the frontal and axial slices show that the molten copper cooled rapidly on the surface, so as to form a copper crust. The cooling was clearly rapid enough to prevent escape of gas bubbles to the top surface (Figure. $4 d$ and e).

The particularly large pore on the left (Figure. $4 d$ ) has an interesting shape that may be explained in a manner consistent with fast cooling. The gas that formed this blister became trapped between the rapidly solidifying top which is in contact with air and side surfaces and deviating towards the remaining hot molten part that remained towards the right and middle of the ingot. Rapid cooling of the ingot is further supported by the formation of the central protrusion. The tomography evidence thus indicates that poured molten metal could not properly mix due to rapid cooling in the peripheral region. The high rate of cooling of the molten copper may be a result of the flat shape of the object, especially near the circumference, and may also point to the use of high purity copper (100\% wt copper). Based on an analysis of neutron tomography axial slices, approximately $4 \%$ of ingot consists of corrosion products. In this experiment the tomograms were not able to reveal the thin cuprite layer on the surface of ingot which is clearly visible in the small cross section under binocular microscope which was discussed elsewhere. 


\subsection{Coiled ring}
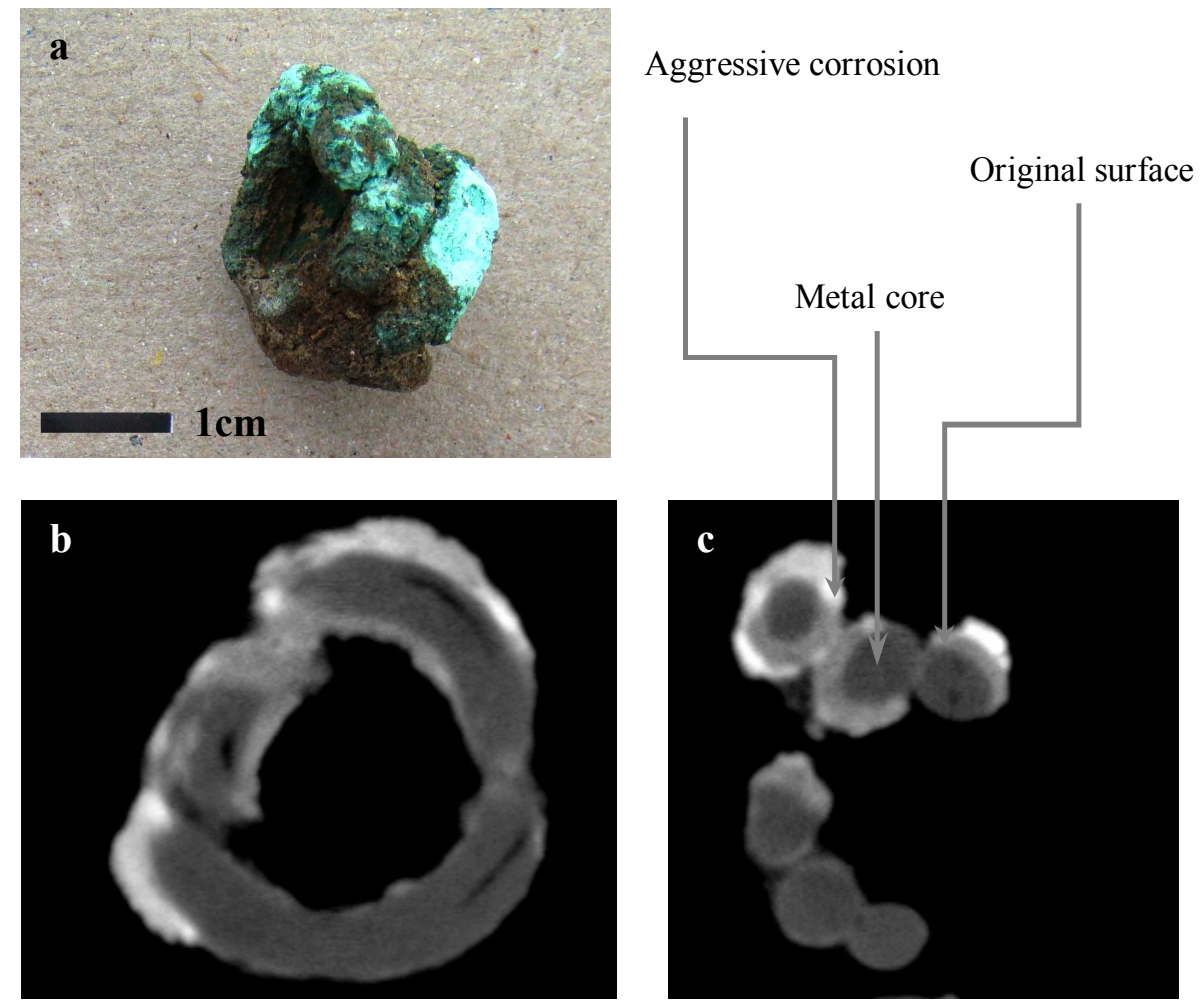

Figure 5. (a) Coiled copper ring from Mapungubwe (b) Frontal neutron tomogram slice showing a discontinues spacing between wires (c) Saggital neutron tomogram slice revealing the lamellar structure of the corrosion layer.

This copper ring (Figure. 5a) was found at Mapungubwe in 1940 by Gardner at a depth of almost $3.5 \mathrm{~m}$ from the surface. It was made of a wire with thickness of 3.79 $\mathrm{mm}$ which was coiled to form a finger ring. Superficial examination indicated that the ring suffers from bronze disease.

A frontal neutron tomogram slice revealed a discontinuous line along the wire strands that can be a sign of strip drawing as wire fabrication method (Figure. 5b). A sagittal neutron tomogram slice (Figure.5c) shows a corrosion layer with a lamellar structure. The corrosion layer can clearly be distinguished from the metal as a result of different neutron attenuation coefficients and density. Although aggressive corrosion disintegrated the original surface, it remained intact in most parts among corrosion. Based on axial tomography slices, $34 \%$ of the sample consists of corrosion products. 

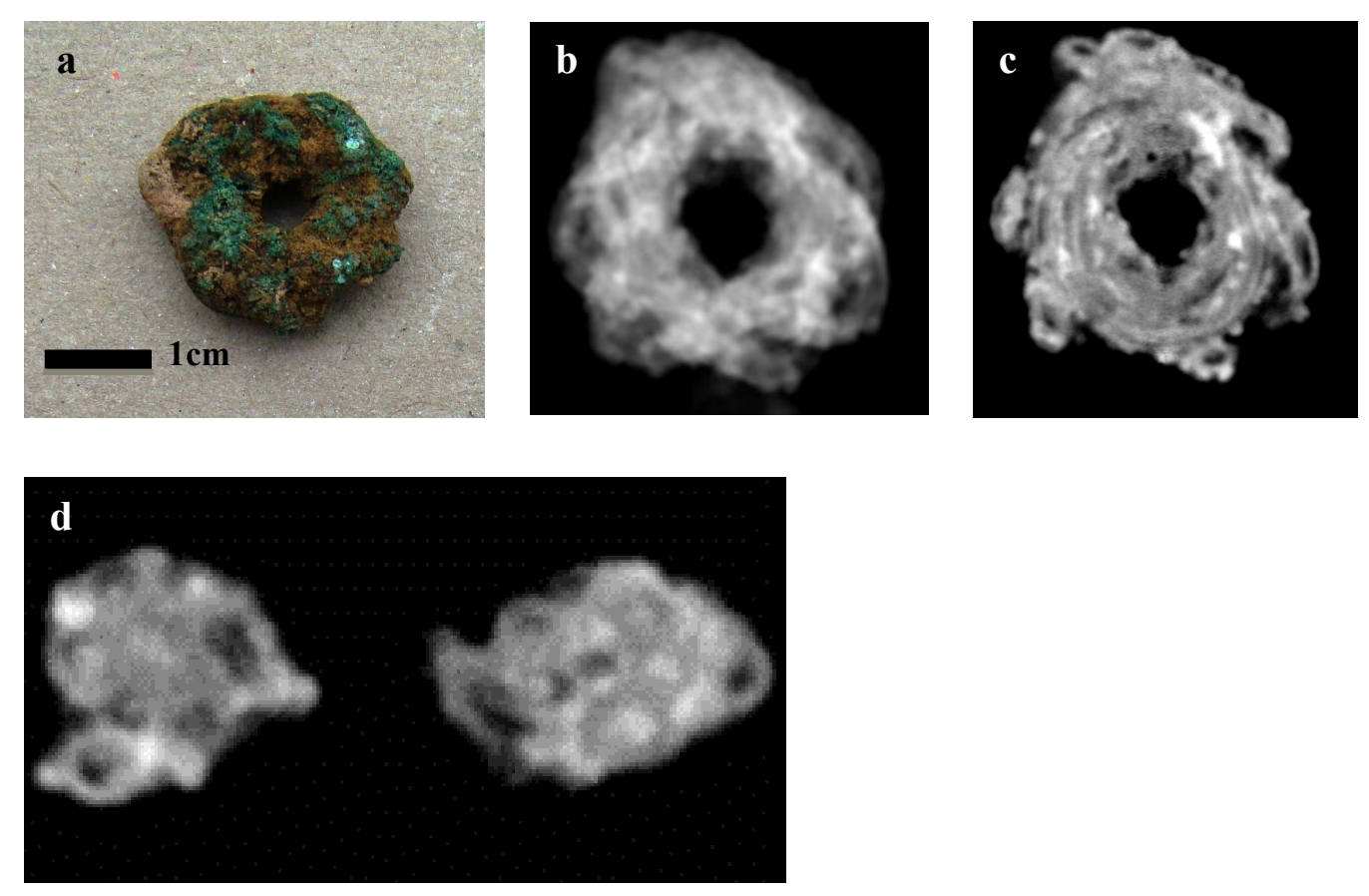

Figure 6. (a) Helically wounded finger ring (b) 2D neutron transmission radiograph almost flattens details (c) A saggital slice revealing the presence of hollows among the wires, corrosion products as bright white areas and original winding path in dark gray. (d) Frontal slice revealing the cross section of the ring and the presence of a core region inside the wire that remains intact only inside some strip wires.

\subsection{Helically wound ring}

The helically wound ring artefact (Figure. 6a) was found in 1971 at Mapungubwe Southern Terrace. Its weight is $4.5 \mathrm{~g}$ and its diameter is almost $21 \mathrm{~mm}$. The wound wire corroded heavily but has kept its original form in amongst the corrosion products. Visual inspection indicates that the ring was probably manufactured by helical twisting of a number of single strip twisted wires (Figure. 6a). It is not clear from visual inspection of the highly corroded ring how many strands of strip twisted wire was used in the construction.

Neutron radiography was performed on this artefact to explore its ability to reveal internal structures of heavily corroded artefacts. Neutron 2D transmission radiographs flattened all the details and corrosion products along with the structure of peripheral wires are only superficial observable (Figure.6b). As expected neutron tomography slices were more revealing. In a sagittal section (Figure.6c) the end sections of some of the strip twisted wire strands can be seen around the inner and outer perimeter (best 
visible around the outside perimeter). Some of the wires are cut more perpendicular to their length axis, thereby giving a roughly circular appearance with the core region visible at the centre, whilst others are cut at more oblique angles, indicating variation in winding pitch. A core, which is a typical element of the structure of strip twisted wires, is distinguishable only inside some wires. Others appear to be hollow. The overall annular structure with a central core region however is strongly indicative of strip wire usage in its construction. In the few wires where a core seems to be present, it is visible due to a higher neutron attenuation coefficient material compared to the peripheral corroded wire but the exact material or detailed structure thereof is not identifiable (Figure.6c and d). It may be a fibrous core alone or a fibrous core with finer iron wire strands incorporated into the fibre bundle as these are two common cores in fabrication of strip twisted wires from Mapungubwe [12]. Corrosion fused all the wires together and in some parts completely destroyed the wire walls. Although no metal core has remained, the overall circular structure of the central region as revealed in (Figure. 6d) strongly suggests the original presence of such a structure. Due to the complex geometry and highly inhomogeneous nature of corrosion products, the percentage corrosion could not be determined. From appearance of the neutron images alone a general conclusion that the object is fragile with very little physical stability can nevertheless be drawn.

\subsection{Two fused spatula}

The spatulas (Figure. 7a) were found during excavation of MK1 at Mapungubwe in 1973. These spatulas were incrusted together inside a mixture of soil, corrosion products and charcoal that forms a bulky mass. Neutron radiography revealed the spatulas to be in an unstable physical condition but could in fact allow virtual separation. Visualization revealing that one spatula has an intact shaft and a part of the blade missing while in the other one the end part of the shaft is missing. Both of the objects have kept their original form inside the crust and did not become physically consolidated or amalgamated (Figure. $7 b$ ). The area where the two spatulas are in close contact appears bright gray in (Figure. 7b) while the darker regions indicate the presence of an intrusion of a mixture of soil and corrosion products between them. The remaining shaft has a delicate loop on the top (Figure. 7c). A thin wire is twisted along the length that is visible as a screw-like feature in this part 

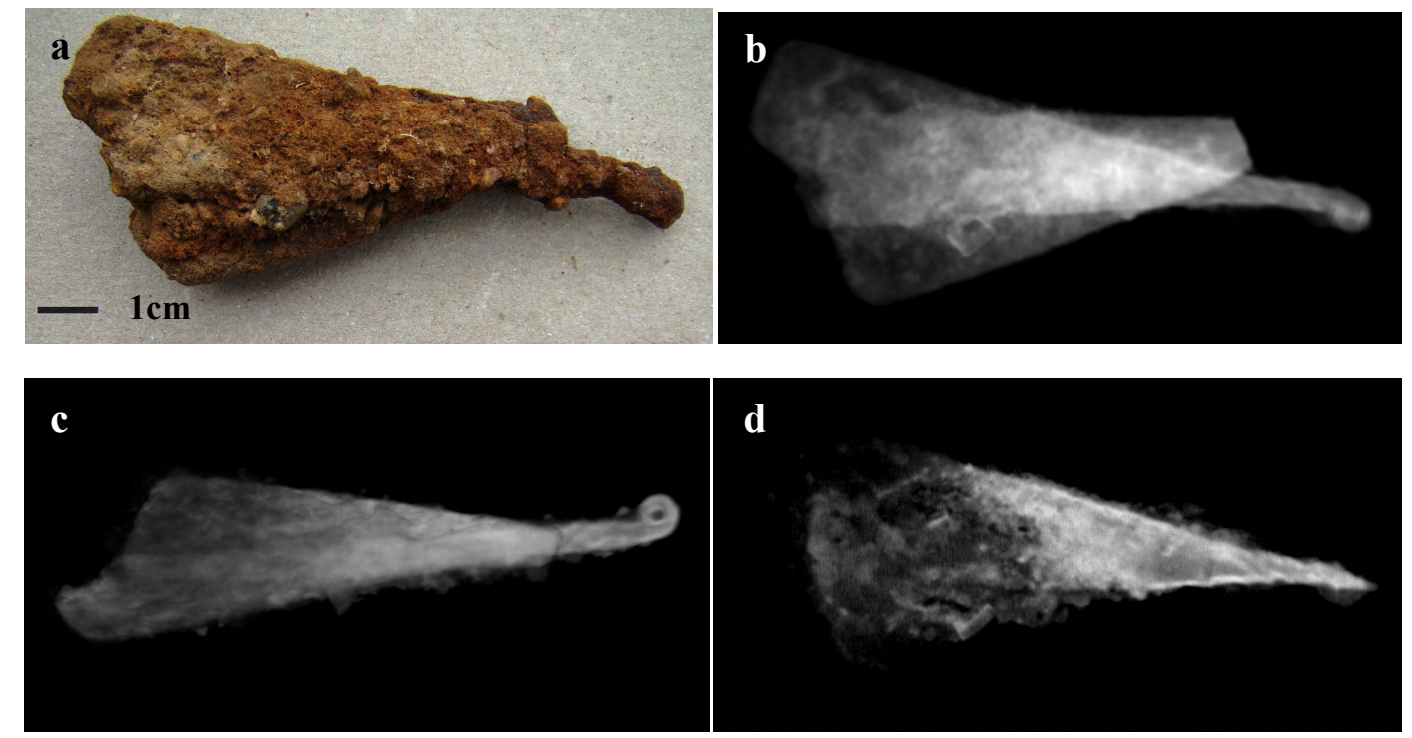

Figure 7: (a) Spatulas encrusted together in a thick layer of sand, charcoal and corrosion products. (b) 2D image showing the general shape of the spatulas (c) 3D results showing that the shaft has a loop. (d) Twisted wire around the shaft is observable in a frontal slice as well as the remainder of the metal core in medium gray.

(Figure. $7 c$ and $d$ ). The low interaction of neutrons with unconsolidated soil allowed the structures inside the crusted clump to be studied in detail, revealing the structure of the corroded artefacts.

Only a small amount of metal core has remained in the shaft, while within the blades no remnant of metal is detected (Figure.7d). Reliable estimation of corrosion percentage was unsuccessful in this case due to a large variation in thickness especially in the fused part.

\section{Conclusions}

The radiography results clearly revealed the complementary nature of X-ray and neutron radiography techniques. Whereas the metal core in the small spatula could be seen best with X-rays, the larger objects required neutron imaging for proper evaluation as a result of the opacity of the heavy metal components to soft energy Xrays (100 $\mathrm{kV}$ and lower). Neutrons also allowed corrosion products to be visualised 
best. Both techniques therefore have a place in artefact studies but for large metal artefacts and details of highly corroded structures neutrons hold a distinct advantage.

The fundamentally different transmission properties of neutrons allow neutron radiography and -tomography to be an excellent qualitative and quantitative diagnostic tool for artefact investigation. The results also conclusively showed the advantage of full 3-D neutron tomography above 2D radiography for more complicated geometry elucidation by producing accurate three dimensional images of objects, from which virtual cuts through any required plane can be visualised. The advantages of neutron tomography as gleaned from this study can be summarised as follows:

1. It is a reliable non-destructive analytical method that allows quantitative evaluation of material fractions due to the accurate area and volume assessment capabilities once the 3D virtual object has been compiled. Defects such as cracks and pores as well as traces of manufacture are easily recognized via neutron $3 \mathrm{D}$ tomograms whereas they are often missed in $2 \mathrm{D}$ radiographs.

2. A survey on a $3 \mathrm{D}$ reconstructed image or tomogram of the object clearly elucidates the exact position of the defect in 3D space.

3. In both low and high thickness metal artefacts, accurate neutron radiographs and tomograms can be achieved.

4. Different composition of corrosion products with their various attenuation coefficients is recognizable on the surface of the objects. In the case of copper where the corrosion layer usually has a lamellar structure this phenomenon is observable.

5. Quantitative estimation of the approximate amount of corrosion products, based on adjusting the contrast of the individual slices of the tomogram to separate the metal zone from corrosion products, is possible provided that the corrosion products contain hydrogen or that other high neutron attenuating elements are present. 
The advantages listed above made it possible to retrace the fabrication methods used in heavily corroded and encrusted artefacts and to also generate vital information needed for proper conservation and maintenance planning.

\section{Acknowledgments}

The authors would like to thank the scientific staff of the SANRAD facility at Necsa for their expert and dedicated collaboration and advice during this study.

\section{References}

[1] A. Meyer, The archaeological sites of Greefswald Stratigraphy and chronology of the sites and a history of investigations, University of Pretoria, Pretoria, 1998.

[2] N. Kardjilov, F. Fiori, G.Giunta, A.Hilger, F. Rustichelli, M. Strobl, Neutron Tomography for Archaeological Investigations, Journal of Neutron Research , 14 (1) (2006) 29-36.

[3] E. Deschler-Erb, E. H. Lehmann, L. Pernet, P. Vontobel, S. Hartmann, The complementary use of neutrons and X-rays for the non-destructive investigation of arcaeological objects from Swiss collections. Archaeometry , 46 (4) (2004) 647-661.

[4] S. D. Bonadies, Tomography of Ancient Bronzes, In: D. A. Scott, J. Podany, B. B. Considine (Eds.), Ancient and Historc metals: Conservation and Scientific Research Part 1 of 3, Proceedings of a symposium organized by the J.Paul Getty Museum and the Getty Conservation Institute, The Getty Conservation Institute, 1991, pp. 75-83.

[5] Y. Cheng, J. Mishara, A Computerized Axial Tomographic Study of Museum Objects, in: E.V. Sayre, P.B. Vandiver, J. Druzik, C. Stevenson (Eds), Materials Issues in Art and Archaeology, Materials Research Societty Symposium Proceedings 123, 1988, pp.19-38.

[6] B. Schillinger, R. Gebhard, B.Haas, W. Ludwig, C. Rausch, U. Wagner, Proceedings of the Fifth World Conference on Neutron Radiography, Berlin, 1996, p. 688.

[7] J. J. Rant, Z. Milic, P. Turk, I. Lengar, Neutron Radiograhy as a NDT method in Archaeology, The 8th International Conference of the Slovenian Society for NonDestructive Testing Application of Contemporary Non-Destructive Testing in engineering, Portoroz, Slovenia, 2005, pp. 181-188.

[8] P. Kudejova, Neutron and X-ray Imaging of the Black Boxes For the ancient Charm Project, Archeometriai Mühely 1(2008) 35-40. 
[9] F. C. de Beer, H. Botha, E. Ferg, R. Grundlingh, A. Smith, Archaeology benefits from neutron tomography investigations in South Africa, Nuclear Instruments and Methods in Physics Research A 605 (2009) 167-170.

[10] E. H. Lehmann, E. Deschler-Erb, A. Ford, Neutron tomography as a valuable tool for the non-destructive analysis of historical bronze sculptures, Archaeometry 52 (2) (2010) 272-285.

[11] F.C. de Beer, Characteristics of the neutron/X-ray tomography system at the SANRAD facility in South Africa, Nuclear Instruments and Methods in Physics Research A 542 (2005) 1-8.

[12] D. Miller, Metal assemblages from Greefswald areas K2, Mapungubwe Hill and Mapungubwe Southern Terrace, South African Archaeological Bulletin 56 (2001) 83103. 